\title{
3D homogenized limit analysis of masonry buildings under horizontal loads
}

\author{
Gabriele Milani $^{\mathrm{a}, *}$, Paulo Lourenço ${ }^{\mathrm{b}}$, Antonio Tralli $^{\mathrm{a}}$ \\ ${ }^{a}$ Department of Engineering, University of Ferrara, via Saragat 1, 44100 Ferrara, Italy \\ ${ }^{\mathrm{b}}$ Department of Civil Engineering, University of Minho, School of Engineering, 4800-058 Azurem, Guimarães, Portugal
}

Received 25 May 2006; received in revised form 19 December 2006; accepted 2 March 2007

Available online 8 April 2007

\begin{abstract}
The current confidence levels in the ability to provide buildings with adequate resistance to horizontal actions do not easily apply to historic and existing masonry structures. Limit analysis is often not sufficient for a full structural analysis under seismic loads, but it can be profitably used in order to obtain a simple and fast estimation of collapse loads. Often, the limit analysis of ancient masonry structures is used in the context of several simplifications, the assumptions about the collapse mechanisms being the most relevant. Aiming at a more general framework, a micromechanical model developed previously by the authors for the limit analysis of isolated in- and out-of-plane loaded masonry walls is extended here and utilized in the presence of coupled membrane and flexural effects. In the model, the elementary cell is subdivided along its thickness in several layers, where fully equilibrated stress fields adopting a polynomial expansion are assumed. The continuity of the stress vector on the interfaces between adjacent sub-domains and anti-periodicity conditions on the boundary surface are further imposed. Linearized homogenized surfaces for masonry in six dimensions are obtained and implemented in a FE limit analysis code, and two 3D case studies are analyzed making use of the kinematic theorem of limit analysis. From the results, the approach proposed is validated and its usefulness for solving engineering problems is demonstrated.
\end{abstract}

(C) 2007 Elsevier Ltd. All rights reserved.

Keywords: Masonry; Limit analysis; Kinematic approach; 3D analysis

\section{Introduction}

It has been shown that the high vulnerability of historical masonry buildings to horizontal actions is mostly due to the absence of adequate connections between the various parts, especially when wooden beams are present both in the floors and in the roof [1]. This characteristic leads to overturning collapses of the perimeter walls under seismic horizontal acceleration and combined in- and out-of-plane failures. The evaluation of the ultimate load bearing capacity of masonry buildings subjected to horizontal loads is a fundamental task in their design and safety assessment. Simplified limit analysis methods are usually adopted by practitioners for safety analyses and design of strengthening [2]. However, codes of practice, such as for instance the recent Italian O.P.C.M. 3431 [3, 4], require a static nonlinear analysis for existing masonry

\footnotetext{
* Corresponding author. Tel.: +390532974911.

E-mail addresses: gmilani@ing.unife.it (G. Milani),pbl@civil.uminho.pt (P. Lourenço), atralli@ing.unife.it (A. Tralli).
}

buildings, in which a limited ductile behavior of the elements is taken into account, featuring failure mechanisms such as rocking, shear and diagonal cracking of the walls. Nowadays, several models for the analysis of masonry buildings are at our disposal, but the approach based on the use of averaged constitutive equations seems to be the only one suitable for employment in a large scale finite element analysis [5]. Heterogeneous approaches based on a distinct representation of bricks and joints seem to be limited to the study of panels of small dimensions, due to the large number of variables involved in a nonlinear finite element analysis. Therefore, alternative strategies based on macro-modeling have been recently developed in order to tackle engineering problems (see Lourenço et al. [6]). Obviously, macro-approaches require a preliminary mechanical characterization of the model, which has to be derived from experimental data from laboratory or in situ testing [7].

In this framework, homogenization techniques can be used for the analysis of large scale structures. Such techniques take into account at a cell level the mechanical properties of 
constituent materials and the geometry of the elementary cell, allowing the analysis of entire buildings through standard finite element codes. Furthermore, the application of homogenization theory to the rigid-plastic case [8] requires only a reduced number of material parameters and provides significant information at failure, such as limit multipliers, collapse mechanisms, and at least on critical sections, the stress distribution [9].

In this paper, the micro-mechanical model presented by the authors in $[9,10]$ and [11] for the limit analysis of in- and out-of-plane loaded masonry walls respectively, is extended and utilized in the presence of coupled membrane and flexural effects. In the model, the elementary cell is subdivided along its thickness into several layers. For each layer, fully equilibrated stress fields are assumed, adopting polynomial expressions for the stress tensor components in a finite number of sub-domains. The continuity of the stress vector on the interfaces between adjacent sub-domains and suitable anti-periodicity conditions on the boundary surface are further imposed. In this way, linearized homogenized surfaces in six dimensions (polytopes) for in- and out-of-plane loaded masonry are obtained. Such surfaces are then implemented in a FE limit analysis code for the analysis at collapse of entire 3D structures, and meaningful examples of technical relevance are discussed in detail.

In Section 2, the micro-mechanical model adopted for obtaining masonry homogenized polytopes is recalled, whereas in Section 3 the FE upper bound approach is presented. The method is based on a triangular discretization of the structure, so that the velocity field interpolation is linear inside each element. Plastic dissipation can occur for in-plane actions both in the continuum and in the interfaces. On the other hand, since the velocities interpolation is assumed linear inside each element, the curvature rate tensor is equal to zero for each triangle, and out-of-plane dissipation can take place only at the interfaces between adjoining triangles.

Two meaningful structural examples are treated in detail in Section 4 . The first numerical simulation refers to the prediction of the ultimate seismic load of a two story masonry building of dimensions $7.32 \times 7.32 \times 7.14 \mathrm{~m}$ (length $\times$ width $\times$ height) The building was experimentally tested by Yi et al. [12] under cyclic loads in the inelastic range at Georgia Tech, USA. The second example consists of an ancient house already studied by De Benedictis et al. in [13] within an extensive survey project coordinated by Giuffrè [2] of the entire Ortigia (Italy) city center.

The reliability of the proposed model is assessed through previously presented numerical results [14], and through comparisons with results obtained by means of standard nonlinear FE approaches.

\section{In- and out-of-plane homogenized failure surfaces}

A masonry wall $\Omega$ constituted by a periodic arrangement of bricks and mortar disposed in running bond texture is considered, as shown in Fig. 1a. As pointed out by Suquet in [8] from a general point of view, homogenization techniques combined with limit analysis can be applied for the evaluation of the homogenized in- and out-of-plane strength domains $S^{\text {hom }}$ of the masonry. Under the assumptions of perfect plasticity and its associated flow rule for the constituent materials, and in the framework of the lower bound limit analysis theorem, $S^{\text {hom }}$ can be derived by means of the following (nonlinear) optimization problem (see also Fig. 1):

$S^{\text {hom }}$

$$
=\left\{\max (\mathbf{M}, \mathbf{N}) \mid\left\{\begin{array}{l}
\mathbf{N}=\frac{l}{|Y|} \int_{y \times h} \sigma \mathrm{d} V \\
\mathbf{M}=\frac{l}{|Y|} \int_{Y \times h} y_{3} \sigma \mathrm{d} V \\
\operatorname{div} \boldsymbol{\sigma}=\mathbf{0} \\
{[[\boldsymbol{\sigma}]] \mathbf{n}^{\text {int }}=\mathbf{0}} \\
\boldsymbol{\sigma} \mathbf{n} \text { anti-periodic on } \partial Y_{l} \\
\boldsymbol{\sigma}(\mathbf{y}) \in S^{m} \forall \mathbf{y} \in Y^{m} ; \boldsymbol{\sigma}(\mathbf{y}) \in S^{b} \forall \mathbf{y} \in Y^{b}
\end{array}\right.\right.
$$

where:

- $\mathbf{N}$ and $\mathbf{M}$ are the macroscopic in-plane (membrane forces) and out-of-plane (bending moments and torsion) tensors;

- $\sigma$ denotes the microscopic stress tensor;

$-\mathbf{n}$ is the outward versor of $\partial Y_{l}$ surface, Fig. 1a;

$-\partial Y_{l}$ is defined in Fig. 1a;

- $[[\sigma]]$ is the jump of micro-stresses across any discontinuity surface of normal $\mathbf{n}^{\text {int }}$, Fig. 1c;

$-S^{m}$ and $S^{b}$ denote respectively the strength domains of mortar and bricks;

$-Y$ is the cross-section of the 3D elementary cell with $y_{3}=0$ (see Fig. 1) $|Y|$ is its area, $V$ is the elementary cell volume, $h$ represents the wall thickness, and $\mathbf{y}=\left(\begin{array}{lll}y_{1} & y_{2} & y_{3}\end{array}\right)$ are the assumed material axes;

- condition (1(c)) imposes a micro-equilibrium with zero body forces, usually neglected in the framework of the homogenization theory;

- anti-periodicity condition (1(e)) requires that the stress vectors $\boldsymbol{\sigma} \mathbf{n}$ are opposite on opposite sides of $\partial Y_{l}$, Fig. 1c, i.e. $\sigma^{(m)} \mathbf{n}_{1}=-\sigma^{(n)} \mathbf{n}_{2}$;

$-Y^{m}$ and $Y^{b}$ represent mortar joints and bricks respectively, see Fig. 1.

In order to solve Eq. (1) numerically, the simple admissible and equilibrated micro-mechanical model proposed in [10] is adopted. The unit cell is subdivided into a fixed number of layers along its thickness, as shown in Fig. 1b. For each layer, out-of-plane components $\sigma_{i 3}(i=1,2,3)$ of the micro-stress tensor $\sigma$ are set to zero, so that only in-plane components $\sigma_{i j}(i, j=1,2)$ are considered active. Furthermore, $\sigma_{i j}(i, j=$ $1,2)$ are kept constant along the $\Delta_{L}$ thickness of each layer, i.e. in each layer $\sigma_{i j}=\sigma_{i j}\left(y_{1}, y_{2}\right)$. For each layer, onefourth of the representative volume element is sub-divided into nine geometrical elementary entities (sub-domains), so that the entire elementary cell is sub-divided into 36 sub-domains (see [10] for further details and Fig. 1b).

For each sub-domain $(k)$ and layer $(L)$, polynomial distributions of degree $(m)$ in the variables $\left(y_{1}, y_{2}\right)$ are a priori assumed for the stress components. Since the stresses are polynomial expressions, the generic $i j$ th component can be written as follows:

$\sigma_{i j}^{(k, L)}=\mathbf{X}(\mathbf{y}) \mathbf{S}_{i j}^{(k, L) T} \quad \mathbf{y} \in Y^{(k, L)}$ 


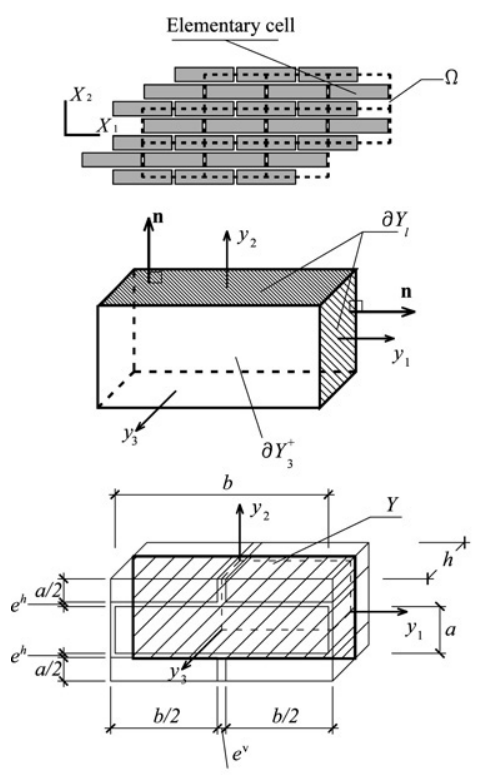

a

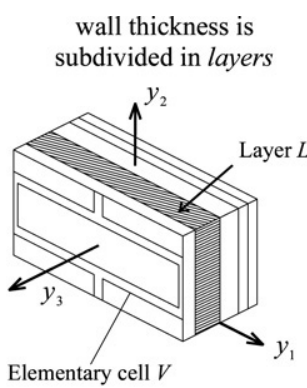

Each layer is subdivided in 36 sub-domains

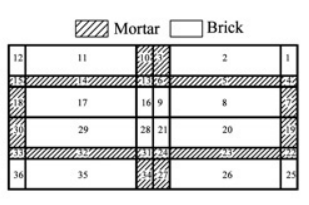

b

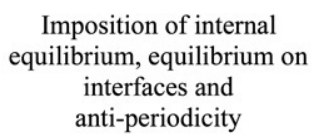

Imposition of internal

equilibrium, equilibrium on interfaces and anti-periodicity
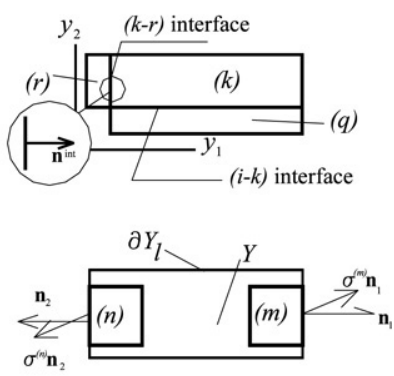

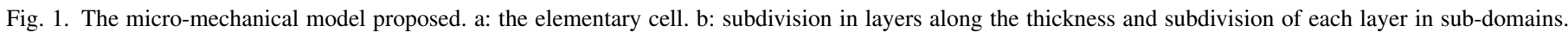
c: imposition of internal equilibrium, equilibrium on interfaces, and anti-periodicity.

where:

$-\mathbf{X}(\mathbf{y})=\left[\begin{array}{llllll}1 & y_{2} & y_{1}^{2} & y_{1} y_{2} & y_{2}^{2} & \ldots\end{array}\right]$

$-\mathbf{S}_{i j}^{(k, L)}=\left[\begin{array}{lll}S_{i j}^{(k, L)(1)} & S_{i j}^{(k, L)(2)} & S_{i j}^{(k, L)(3)}\end{array} S_{i j}^{(k, L)(4)} S_{i j}^{(k, L)(5)}\right.$

$\left.S_{i j}^{(k, L)(6)} \quad \ldots\right]$ is a vector representing the unknown stress parameters of sub-domain $(k)$ of layer $(L)$;

$-Y^{(k, L)}$ represents the $k$ th sub-domain of layer $(L)$.

The imposition of equilibrium inside each sub-domain, the continuity of the stress vector on the interfaces, and the antiperiodicity of $\boldsymbol{\sigma} \mathbf{n}$ permit a strong reduction in the number of independent stress parameters. For the sake of conciseness, the reader is referred to [10] for further details.

Elementary assemblage operations on the local variables allow us to write the stress vector $\tilde{\boldsymbol{\sigma}}^{(k, L)}$ of layer $L$ inside each sub-domain as:

$\widetilde{\boldsymbol{\sigma}}^{(k, L)}=\widetilde{\mathbf{X}}^{(k, L)}(\mathbf{y}) \widetilde{\mathbf{S}}^{(L)} \quad k=1, \ldots$, no. of sub-domains

$L=1, \ldots$, no. of layers

where $\widetilde{\mathbf{S}}^{(L)}$ is a $N_{\mathrm{uk}} \times 1\left(N_{\mathrm{uk}}=\right.$ number of unknowns per layer $)$ vector of linearly independent unknown stress parameters of layer $L$ and $\widetilde{\mathbf{X}}^{(k, L)}(\mathbf{y})$ is a $3 \times N_{\mathrm{uk}}$ matrix depending only on the geometry of the elementary cell and on the position $\mathbf{y}$ of the point in which the micro-stress is evaluated.

As already pointed out, once an equilibrated polynomial field in each layer is obtained, the proposed in- and out-ofplane model requires a subdivision $\left(n_{L}\right)$ of the wall thickness into several layers (Fig. 1b), with a fixed constant thickness $\Delta_{L}=h / n_{L}$ for each layer. This allows us to derive the following simple (non) linear optimization problem:

$$
\begin{aligned}
& S^{\text {hom }} \\
& \text { such that }\left\{\begin{array}{l}
\max \{\lambda\} \\
\tilde{\mathbf{N}}=\int_{k, L} \tilde{\boldsymbol{\sigma}}^{(k, L)} \mathrm{d} V \\
\tilde{\mathbf{M}}=\int_{k, L} y_{3} \widetilde{\boldsymbol{\sigma}}^{(k, L)} \mathrm{d} V \\
\boldsymbol{\Sigma}=[\widetilde{\mathbf{N}} \widetilde{\mathbf{M}}]=\lambda \mathbf{n}_{\Sigma} \\
\tilde{\boldsymbol{\sigma}}^{(k, L)}=\widetilde{\mathbf{X}}^{(k, L)}(\mathbf{y}) \widetilde{\mathbf{S}} \\
\tilde{\boldsymbol{\sigma}}^{(k, L)} \in S^{(k, L)} \\
k=1, \ldots, \text { number of sub-domains } \\
L=1, \ldots, \text { number of layers }
\end{array}\right.
\end{aligned}
$$

where:

$-\lambda$ is the load multiplier (ultimate moment, ultimate membrane action, or a combination of moments and membrane actions) with fixed direction $\mathbf{n}_{\Sigma}$ in the six dimensional space of membrane actions $(\tilde{\mathbf{N}}=$ $\left.\left[\begin{array}{lll}N_{x x} & N_{x y} & N_{y y}\end{array}\right]\right)$ and bending + torsion moments $(\tilde{\mathbf{M}}=$ $\left[\begin{array}{lll}M_{x x} & M_{x y} & M_{y y}\end{array}\right]$.

- $S^{(k, L)}$ denotes the (nonlinear) strength domain of the constituent material (mortar or brick) corresponding to the $\underset{\sim}{\mathrm{S}}$ th sub-domain and $L$ th layer.

- $\widetilde{\mathbf{S}}$ collects all the unknown polynomial coefficients (of each sub-domain of each layer).

It is noted that the direction $\mathbf{n}_{\Sigma}$ is fixed arbitrarily in six dimensional space $[\tilde{\mathbf{N}} \tilde{\mathbf{M}}]$. As a rule, since $\mathbf{n}_{\Sigma}=$ $\left[\alpha_{1}, \alpha_{2}, \ldots, \alpha_{6}\right]$ with $\Sigma \alpha_{i}^{2}=1$, the parameteres $\alpha_{i}$ are chosen randomly between 0 to 1 sastisfying the constraint $\Sigma \alpha_{i}^{2}=$ 1 , so that a number of directions $\mathbf{n}_{\Sigma}$ are selected. For each direction, a linear programming problem is solved and a point of the failure surface obtained. By means of the points obtained through such a process, a Delaunay tessellation is performed, 


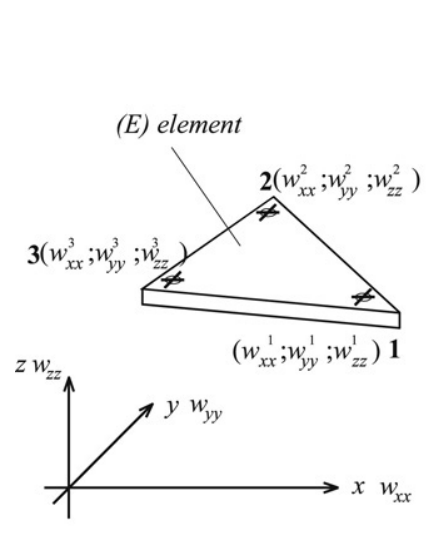

a

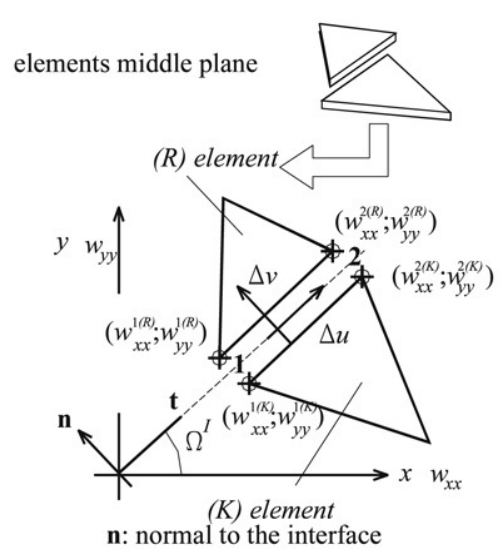

b

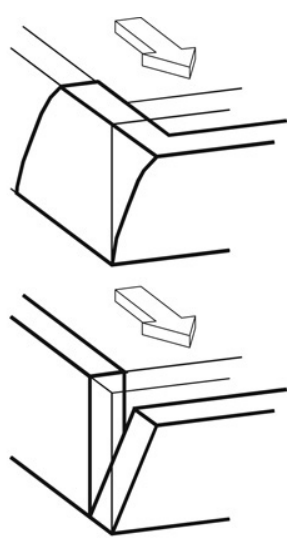

C

Fig. 2. a: Triangular plate and shell element used for the upper bound FE limit analysis. b: discontinuity of the in-plane velocity field. c: perfect interlocking and absence of connections between perpendicular walls.

and a lower bound linear approximation of the failure surface is recovered.

In what follows, the wall thickness is subdivided into thirty layers, a subdivision which represents a marginal processing time and should be compared with the typical less than ten thickness integration points in FE analysis using layered shell elements. The authors found that more refined discretizations resulted in negligible changes in the homogenized failure surface.

It is worth noting that the model at hand is able to reproduce the typical anisotropic behavior of masonry at failure, as well as a no tension material if a Mohr-Coulomb failure criterion with zero cohesion is assumed for joints and vertical compressive stresses are not present.

\section{3D kinematic FE limit analysis: Basic assumptions}

The upper bound approach developed in this paper is based both on the formulation presented in [15] by Sloan and Kleeman for the in-plane case and on the formulation by Munro and Da Fonseca [16] for out-of-plane actions, also adopted in [17].

The formulation uses three-noded (triangular) elements with linear interpolation of the velocity field inside each element. In addition, for the in-plane case, discontinuities of the velocity field along the edges of adjacent triangles are introduced. It has been shown $[9,15]$, in fact, that the definition of kinematically admissible velocity fields with discontinuities on interfaces is adequate for purely cohesive or cohesive-frictional materials, which is the case of masonry.

On the other hand, it is stressed that an important limitation of the limit analysis approach adopted here is its inability to give information of the displacements at collapse (information required by some codes of practice). Moreover, an infinite plastic deformation capacity of the material is assumed: this hypothesis should be checked case by case, depending on the geometry of the masonry wall and on the distribution of loads applied.

\subsection{Kinematic constraints and plastic flow relationships}

Three velocity unknowns per node $i$, say $w_{x x}^{i}, w_{y y}^{i}$, and $w_{z z}^{i}$ (respectively 2 in-plane velocities and 1 out-of-plane velocity, see Fig. 2a) are introduced for each element $E$, so that the velocity field is linear inside an element, whereas the strain rate field is constant for in-plane actions.

For the sake of simplicity, it is assumed that any jump of velocities on interfaces occurs only in the plane containing two contiguous and coplanar elements, with linear interpolation of the jump along the interface. Hence, for each interface between coplanar adjacent elements, four additional unknowns are introduced $\left(\Delta \mathbf{u}^{I}=\left[\begin{array}{llll}\Delta v_{1} & \Delta u_{1} & \Delta v_{2} & \Delta u_{2}\end{array}\right]^{\mathrm{T}}\right)$, representing the normal $\left(\Delta v_{i}\right)$ and tangential $\left(\Delta u_{i}\right)$ jumps of velocities (with respect to the discontinuity direction) evaluated on nodes $i=1$ and $i=2$ of the interface (see Fig. 2b). For the sake of simplicity, it is assumed in the model that, if two adjacent elements do not lie in the same plane, no discontinuity occurs between the velocities belonging to the elements, so a priori we assume a perfect interlocking between perpendicular walls (see Fig. 2c). This interlocking can be controlled indirectly by the horizontal masonry tensile strength or forced equal to zero, in case of in situ evidence after a survey of the building.

Hence, for any pair of nodes on the interface between two adjacent and coplanar triangles $R$ and $K$, the tangential and normal velocity jumps can be written in terms of the Cartesian nodal velocities of elements $R-K$ (see [15] for details), so that four linear equations in the following form can be written:

$\mathbf{A}_{11}^{\mathrm{eq}} \mathbf{w}^{R}+\mathbf{A}_{12}^{\mathrm{eq}} \mathbf{w}^{K}+\mathbf{A}_{13}^{\mathrm{eq}} \Delta \mathbf{u}^{I}=\mathbf{0}$

where $\mathbf{w}^{R}$ and $\mathbf{w}^{K}$ are the $9 \times 1$ vectors that collect velocities of elements $R$ and $K$ respectively, and $\mathbf{A}_{1 j}^{\mathrm{eq}} j=1,2,3$ are matrices which depend only on the interface orientation $\Omega^{I}$ (Fig. 2).

For a continuum under in-plane loads, three equality constrains representing the plastic flow in the continuum (obeying an associated flow rule) are introduced for each 
element:

$\dot{\varepsilon}_{\mathrm{pl}}^{E}=\left[\frac{\partial u_{x x}}{\partial x} \frac{\partial u_{y y}}{\partial y} \frac{\partial u_{y y}}{\partial x}+\frac{\partial u_{x x}}{\partial y}\right]=\dot{\lambda}^{E} \partial S^{\text {hom }} / \partial \boldsymbol{\Sigma}$

where:

$-\dot{\boldsymbol{\varepsilon}}_{\mathrm{pl}}^{E}$ is the plastic strain rate vector of element $E$;

$-\dot{\lambda}^{E} \geq 0$ is the plastic multiplier;

$-S^{\text {hom }}$ is the homogenized (non) linear failure polytope of masonry;

$-\Sigma$ is the vector of macroscopic variables $\boldsymbol{\Sigma}=$ $\left(N_{11}, N_{12}, N_{22}, M_{11}, M_{12}, M_{22}\right)$.

It is worth noting that out-of-plane components of the plastic strain rate are $\dot{\varepsilon}_{\mathrm{pl}, x z}=\dot{\varepsilon}_{\mathrm{pl}, y z}=0$, whereas $\dot{\varepsilon}_{\mathrm{pl}, z z}$ is constant.

We refer the reader to the previous section and to [9] for further details on the procedure used for obtaining a linear approximation (with $m$ hyper-planes) of the failure polytope in the form $S^{\text {hom }} \equiv \mathbf{A}^{\text {in }} \boldsymbol{\Sigma} \leq \mathbf{b}^{\text {in }}$, where $\mathbf{A}^{\text {in }}$ is a $m \times 6$ matrix of coefficients of each hyper-plane and $\mathbf{b}^{\text {in }}$ is a $m \times 1$ vector of the right hand sides of the linear approximation.

The three linear equality constraints per element can be written in the form $\mathbf{A}_{11}^{\mathrm{eq}} \mathbf{w}^{E}+\mathbf{A}_{12}^{\mathrm{eq}} \lambda^{E}=\mathbf{0}$, where $\mathbf{w}^{E}$ is the vector of element velocities and $\dot{\lambda}^{E}$ is a $m \times 1$ vector of plastic multiplier rates (one for each plane of the linearized failure surface).

Following Munro and Da Fonseca [16], out-of-plane plastic dissipation occurs only along each interface $I$ between two adjacent triangles $R$ and $K$ or on a boundary side $B$ of an element $Q$ (see Fig. 3).

Denoting $\mathbf{w}_{z z, E}=\left[\begin{array}{lll}w_{z z}^{i(E)} & w_{z z}^{j(E)} & w_{z z}^{k(E)}\end{array}\right]^{\mathrm{T}}$ as the element $E$ out-of-plane nodal velocities and $\dot{\boldsymbol{\theta}}_{E}=\left[\begin{array}{lll}\dot{\vartheta}_{i}^{E} & \dot{\vartheta}_{j}^{E} & \dot{\vartheta}_{k}^{E}\end{array}\right]^{\mathrm{T}}$ as the side normal rotation rates, $\dot{\boldsymbol{\theta}}_{E}$ and $\mathbf{w}_{z z, E}$ are linked by the compatibility equation (Fig. 3):

$\dot{\boldsymbol{\theta}}_{E}=\mathbf{B}_{E} \mathbf{w}_{z z, E}$

where

$\mathbf{B}_{E}=\frac{1}{2 A_{E}}\left[\begin{array}{ccc}\frac{b_{i} b_{i}+c_{i} c_{i}}{l_{i}} & \frac{b_{i} b_{j}+c_{i} c_{j}}{l_{i}} & \frac{b_{i} b_{k}+c_{i} c_{k}}{l_{i}} \\ \frac{b_{j} b_{i}+c_{j} c_{i}}{l_{j}} & \frac{b_{j} b_{j}+c_{j} c_{j}}{l_{j}} & \frac{b_{j} b_{k}+c_{j} c_{k}}{l_{j}} \\ \frac{b_{k} b_{i}+c_{k} c_{i}}{l_{k}} & \frac{b_{k} b_{j}+c_{k} c_{j}}{l_{k}} & \frac{b_{k} b_{k}+c_{k} c_{k}}{l_{k}}\end{array}\right]$,

with $b_{i}=y_{j}-y_{k}, \quad c_{i}=x_{k}-x_{j}$

and $A_{E}$ is the element area.

\subsection{Internal and external power dissipation}

The total internal power dissipated, $P^{\text {in }}$, is constituted by the power dissipated in the continuum $P_{E}^{\text {in }}$ and the power dissipated in the interfaces $P_{I}^{\text {in }} \cdot P_{E}^{\text {in }}$, and can be evaluated for each triangle $E$ of area $A_{E}$, taking into account that curvature rates $\dot{\chi}_{x x}$, $\dot{\chi}_{x y}, \dot{\chi}_{y y}$ are zero in continuum, so that the flexural part of the model does not dissipate power in the continuum. As the homogenized (linearized) failure surface is constituted by the $m$ hyper-planes of the equation $A_{x x}^{q} N_{x x}+A_{y y}^{q} N_{y y}+A_{x y}^{q} N_{x y}+$
$B_{x x}^{q} M_{x x}+B_{y y}^{q} M_{y y}+B_{x y}^{q} M_{x y}=C_{E}^{q}$, with the subscript $q$ such that $1 \leq q \leq m$, an estimation of $P_{E}^{\text {in }}$ can be obtained as follows:

$$
\begin{gathered}
P_{E}^{\text {in }}=A_{E} \sum_{q=1}^{m} C_{E}^{q} \dot{\lambda}_{E}^{(q)} \\
\dot{\chi}_{r q}=\sum_{q=1}^{m} \dot{\lambda}_{E}^{(q)} \partial S^{\text {hom }} / \partial \Sigma_{M_{r q}}=0 \\
r q=x x, x y, y y \Sigma_{M_{r q}}=M_{r q}
\end{gathered}
$$

where $\dot{\lambda}_{E}^{(q)}$ is the plastic multiplier rate of the triangle $E$ associated with the $q$ th hyper-plane of the linearized failure surface.

For an interface $I$ of length $\Gamma$ and orientation $\Omega^{I}$, a rotation operator is applied to the linearized homogenized failure surface in order to obtain, with a few row operations, $m$ equations (one for each hyper-plane) in the form $A_{t t}^{q} N_{t t}+$ $A_{n n}^{q} N_{n n}+A_{t n}^{q} N_{t n}+B_{t t}^{q} M_{t t}+B_{n n}^{q} M_{n n}+B_{t n}^{q} M_{t n}=C_{I}^{q}$ representing the homogenized failure surface $\widetilde{S}^{\text {hom }}$ in the $\mathbf{n}-\mathbf{t}$ interface frame of reference, Fig. 2.

Therefore, the power dissipated, $P_{I}^{\text {in }}$, along an interface $I$ of length $\Gamma$ and with orientation $\Omega^{I}$ can be estimated as follows:

$$
\begin{aligned}
& P_{I}^{\text {in }}=\int_{\Gamma} \sum_{q=1}^{m} C_{I}^{q} \dot{\lambda}_{I}^{(q)}(\xi) \mathrm{d} \xi \quad \dot{\vartheta}^{I}=\dot{\vartheta}_{i}^{R}+\dot{\vartheta}_{j}^{K} \\
& \dot{\vartheta}_{n t}^{I}=\sum_{q=1}^{m} \dot{\lambda}_{E}^{(q)} \partial \widetilde{S}^{\mathrm{hom}} / \partial \Sigma_{M_{n t}}=0 \\
& \dot{\vartheta}_{t t}^{I}=\sum_{q=1}^{m} \dot{\lambda}_{E}^{(q)} \partial \widetilde{S}^{\mathrm{hom}} / \partial \Sigma_{M_{t t}}=0 \\
& \Delta_{t t}^{I}=\sum_{q=1}^{m} \dot{\lambda}_{E}^{(q)} \partial \widetilde{S}^{\mathrm{hom}} / \partial \Sigma_{N_{t t}}=0 \\
& \dot{\vartheta}_{n n}^{I}=\sum_{q=1}^{m} \dot{\lambda}_{E}^{(q)} \partial \widetilde{S}^{\mathrm{hom}} / \partial \Sigma_{M_{n n}}=\dot{\vartheta}^{I}
\end{aligned}
$$

where:

$-\dot{\lambda}_{I}^{(q)}(\xi)$ represents the $q$ th plastic multiplier rate of a point $\xi$ of the interface $I$. Assuming the plastic multipliers on the interfaces are linear, Eq. (9) can be written as $P_{I}^{\text {in }}=$ $\Gamma / 2 \sum_{q=1}^{m} C_{I}^{q}\left(\dot{\lambda}_{I 1}^{(q)}+\dot{\lambda}_{I 2}^{(q)}\right)$, where $\dot{\lambda}_{I 1}^{(q)}$ and $\dot{\lambda}_{I 2}^{(q)}$ are the interface plastic multiplier rates of nodes 1 and 2 ;

- $\dot{\vartheta}_{n t}^{I}$ is the torsional rotation rate between $R$ and $K$ along $I$ (see Fig. 3), assumed equal to zero in the model;

- $\dot{\vartheta}_{t t}^{I}$ is the bending rotation rate between $R$ and $K$ on $I$ along t, assumed equal to zero;

- $\Delta_{t t}^{I}$ represents the interface plastic strain rate of $I$ along $\mathbf{t}$, integrated along the infinitesimal thickness of the interface. It is worth noting that this equation involves only the plastic multipliers of the interfaces and is different from the procedure reported in [18], which represents a weak compatibility condition for the interface;

- $\Sigma_{M_{n t}}, \quad \Sigma_{M_{t t}}$, and $\Sigma_{N_{t t}}$ are the torsional moments on $I\left(\Sigma_{M_{n t}}=M_{n t}\right)$, the bending moment perpendicular to $\mathbf{t}$ 

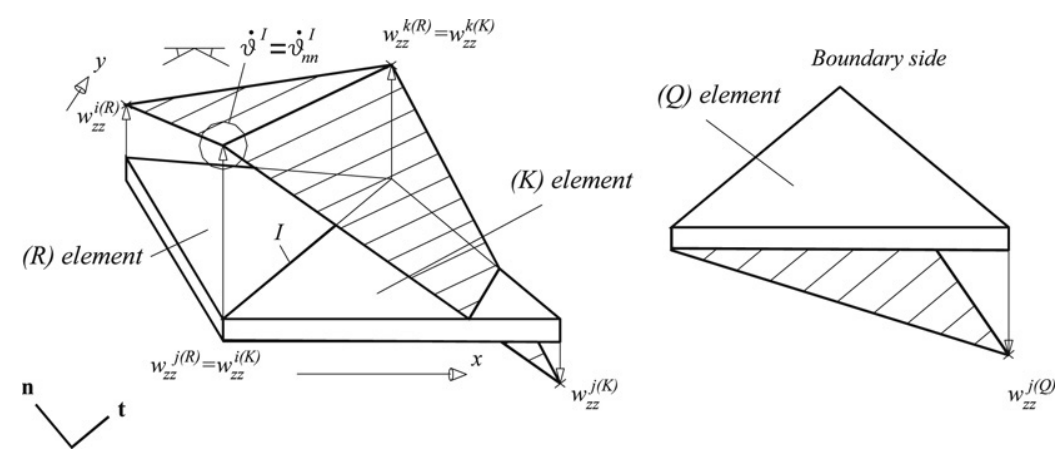

Fig. 3. Rotation along an interface between adjacent triangles or in correspondence of a boundary side.

direction $\left(\Sigma_{M_{t t}}=M_{t t}\right)$ and the in-plane action parallel to $\mathbf{t}\left(\Sigma_{N_{t t}}=N_{t t}\right)$ respectively;

- $\dot{\vartheta}_{n n}^{I}$ is the bending rotation rate between $R$ and $K$ on $I$ along n, i.e. $\dot{\vartheta}^{I}$ (Fig. 3).

External power dissipated can be written as $P^{\mathrm{ex}}=\left(\mathbf{P}+\lambda \mathbf{P}_{1}^{\mathrm{T}}\right) \mathbf{w}$, where $\mathbf{P}_{0}$ is the vector of (equivalent lumped) permanent loads, $\lambda$ is the load multiplier for the structure examined, $\mathbf{P}_{1}^{\mathrm{T}}$ is the vector of (lumped) variable loads, and $\mathbf{w}$ is the vector of assembled nodal velocities. As the amplitude of the failure mechanism is arbitrary, a further normalization condition $\mathbf{P}_{1}^{\mathrm{T}} \mathbf{w}=1$ is usually introduced. Hence, the external power becomes linear in $\mathbf{w}$ and $\lambda$, i.e. $P^{\mathrm{ex}}=\mathbf{P}_{0}^{\mathrm{T}} \mathbf{w}+\lambda$.

\subsection{The linear programming $(L P)$ problem}

After some elementary assemblage operations, a simple linear programming problem is obtained (analogous to that reported in [15]), where the objective function consists in the minimization of the total internal power dissipated:

$$
\left\{\begin{array}{l}
\min \left\{\sum_{I=1}^{n^{I}} P_{I}^{\mathrm{in}}+\sum_{E=1}^{n^{E}} P_{E}^{\mathrm{in}}-\mathbf{P}_{0}^{\mathrm{T}} \mathbf{w}\right\} \\
\text { such that }\left\{\begin{array}{l}
\mathbf{A}^{\mathrm{eq}} \mathbf{U}=\mathbf{b}^{\mathrm{eq}} \\
\dot{\lambda}^{I, \text { ass }} \geq \mathbf{0} \\
\dot{\boldsymbol{\theta}}^{\mathrm{ass}}=\dot{\boldsymbol{\theta}}^{+}-\dot{\boldsymbol{\theta}}^{-} \\
\dot{\boldsymbol{\theta}}^{+} \geq 0
\end{array} \dot{\lambda}^{E, \text { ass }} \geq \mathbf{0}\right.
\end{array}\right.
$$

where:

- $\mathbf{U}$ is the vector of global unknowns and collects the vector of assembled nodal velocities ( $\mathbf{w})$, the vector of assembled element plastic multiplier rates $\left(\dot{\lambda}^{E \text {,ass }}\right)$, the vector of assembled jump of velocities on interfaces $\left(\Delta \mathbf{u}^{I \text {,ass }}\right)$, the vector of assembled interface plastic multiplier rates $\left(\dot{\lambda}^{I, a s s}\right)$, and the vector of interface and boundary out-ofplane rotation angles $\dot{\boldsymbol{\theta}}$.

$-\mathbf{A}^{\mathrm{eq}}$ is the overall constraints matrix and collects normalization conditions, velocity boundary conditions, relations between velocity jumps on interfaces and elements velocities, constraints for plastic flow in velocity discontinuities, and constraints for plastic flow in the continuum.

$-n^{E}$ and $n^{I}$ are the total number of elements and interfaces, respectively.
The reader is referred to [19] and [20] for a critical discussion of the most efficient tools for solving the problem given in Eq. (10).

\section{Structural examples}

In this section, two structural examples are presented, namely a full scale two story building experimentally tested by Yi et al. [12], and an ancient house already studied both by De Benedictis et al. in [13] and by Orduna in [21].

In both the cases presented, a homogenized limit analysis approach is used to predict the ultimate shear at the base for seismic actions and the relevant failure mechanisms. It is worth noting that, for all the cases analyzed, the so called primary collapse mechanisms, as for instance overturnings of single façades, are excluded from imposing perfect interlocking at each corner.

In this way, the limit analysis approach proposed can be compared with standard FE elastic-plastic analyses performed by means of commercially available code (Strand 7). Both failure mechanisms and failure loads show that technically meaningful results can be obtained with the proposed approach.

A sensitivity analysis is also reported, varying in a wide range for both mortar cohesion and mortar friction angle.

It is worth noting that the usefulness of a global limit analysis conducted by means of plate and shell elements on entire buildings stands in its capacity to take into account simultaneously in- and out-of-plane failures, as well as partial collapse mechanisms of single panels. Furthermore, an a priori estimation of the most probable collapse mechanism is not required.

\section{1. $3 D$ limit analysis of a two story unreinforced masonry building}

A 3D FE limit analysis on a two story unreinforced masonry (URM) building experimentally tested by Yi et al. in [12] is considered.

The structure, see Fig. 4, reproduces some structural characteristics of typically existing URM buildings in the midAmerica area. The dimensions of the structure are $7.32 \times 7.32 \mathrm{~m}$ in plan, with story heights of $3.6 \mathrm{~m}$ for the first story and $3.54 \mathrm{~m}$ for the second story. The structure is constituted by four masonry walls labeled walls A, B, 1, and 2, respectively, see 

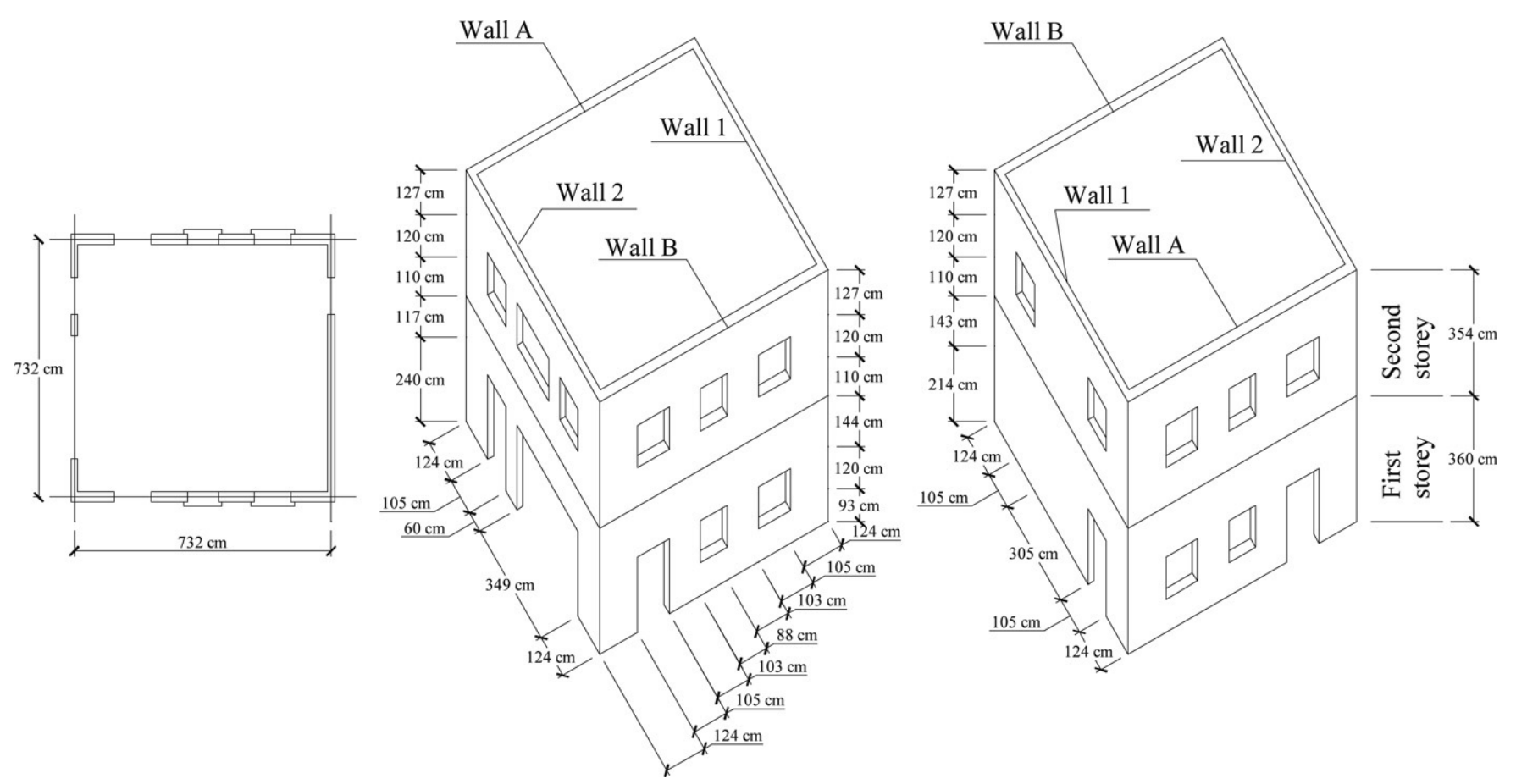

Fig. 4. Yi et al. [12] building, geometry.

Fig. 4. The walls have different thicknesses and opening ratios. Walls 1 and 2 are composed of brick masonry with thickness $20 \mathrm{~cm}$. Wall 1 has relatively small openings, whereas wall 2 contains a large door opening and larger window openings. Therefore, the large difference in stiffness between walls 1 and 2 allows the torsional behavior of the URM building to be investigated. Walls A and B are identical, and with a nominal thickness equal to $30 \mathrm{~cm}$. The moderate opening ratios in these two walls are representative of many existing masonry buildings. The aspect ratios of piers range from 0.4 to 4.0. The four masonry walls are considered perfectly connected at the corners, a feature not always reproduced in the past URM tests. This allows us to investigate also the contribution of the transverse walls to the strength of the overall building.

For walls $\mathrm{A}$ and $\mathrm{B}$, Yi et al. [12] employed masonry arch lintels, whereas for walls 1 and 2, steel lintels were used. A wood diaphragm and a timber roof are present in correspondence of the floors. Both solid bricks and hollowcored bricks are employed in the structure. The nominal dimensions of both types of bricks are $200 \times 89 \mathrm{~mm}$ (length $\times$ width). The cored bricks contain a longitudinal hole through the center with a diameter of $22 \mathrm{~mm}$. Solid bricks are used for the lower 54 courses in the first story of the test structure to approximately the $3.8 \mathrm{~m}$ level, whereas cored bricks are used for the remaining parts of the structure. Vertical loading is constituted only by the wall's self weight, and the permanent loads of the first floor and of the roof.

For the numerical simulations, the masonry density is kept equal to $22.19 \mathrm{kN} / \mathrm{m}^{3}$ [14]. The analysis is conducted using a mesh of 858 triangular elements, Fig. 5a. In order to numerically reproduce the actual experimental set-up, horizontal loads, depending on the limit multiplier, are applied
Table 1

Mechanical characteristics assumed for joints and bricks, Yi et al. [12] masonry building

\begin{tabular}{lll}
\hline Joint & Brick \\
\cline { 1 - 1 } $\begin{array}{ll}c\left(\mathrm{~N} / \mathrm{mm}^{2}\right) \\
\text { cohesion }\end{array}$ & $\begin{array}{l}\Phi \text { friction } \\
\text { angle }\end{array}$ & $f_{c}\left(\mathrm{~N} / \mathrm{mm}^{2}\right)$ compressive strength \\
\hline 0.01 & $\tan ^{-1}(1.0)$ & 10 (solid bricks) 4.1 (hollow bricks) \\
\hline
\end{tabular}

in correspondence to the first and second floor levels of wall 1 , as shown in Fig. 5b. Parameters $\alpha_{i}$ in this figure are taken equal to $W_{i} / \sum_{i}^{2} W_{i}$, where $W_{i}$ is the $i$ th floor vertical load. The masonry self weight, which corresponds to a large percentage of the total gravity load, is supposed for the sake of simplicity, to be concentrated in correspondence to the first and second floors. The mechanical properties of the bricks and mortar are reported in Table 1. Such characteristics are found to be in agreement both with experimental data collected in [12] and the pushover analyses presented in [14]. For the joints, a classic Mohr-Coulomb failure criterion is adopted, whereas a compressive cut-off is adopted for the bricks.

The results obtained with the homogenized FE limit analysis model (i.e. failure shear at the base and failure mechanism) are compared with the experimental force-displacement diagrams in Fig. 6, where the total shears at the base of walls $\mathrm{A}$ and $\mathrm{B}$ are reported. The kinematic FE homogenized limit analysis gives a base shear of $183 \mathrm{kN}$ for walls A and $\mathrm{B}$, respectively, in excellent agreement with the results obtained experimentally [12]. Finally, a comparison between the deformed shape at collapse obtained with the model proposed, Fig. 7a and b, and the deformed shape of the 

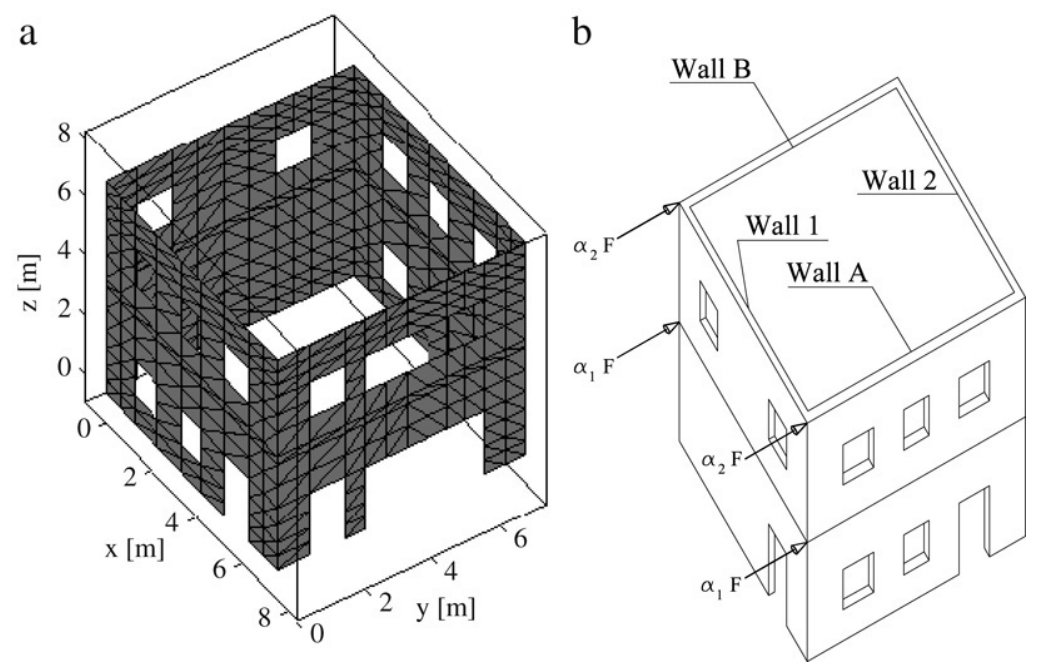

Fig. 5. Yi et al. [12] building. a: mesh used for the limit analysis and b: points of application of horizontal loads.

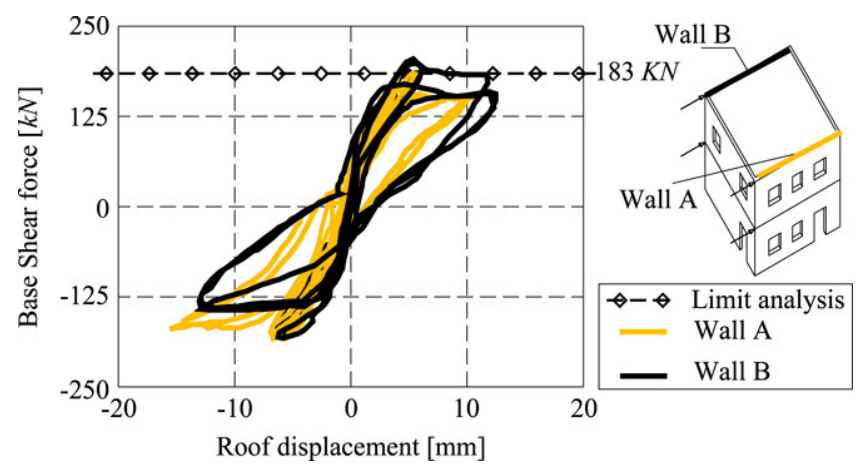

Fig. 6. Yi et al. [12] building. Comparison between force-displacement experimental curves and the numerical collapse load.

model developed in [14], Fig. 7c, shows the accuracy of the homogenized limit analysis presented.

A sensitivity analysis is also conducted on the example at hand. A classic Mohr-Coulomb failure criterion with tension cut-off $f_{t}$ is considered for joints, assuming $f_{t}$ being constantly equal to $\min \left\{0.05 \mathrm{~N} / \mathrm{mm}^{2} c / \tan \Phi\right\}$, and varying the cohesion $c$ and the friction angle $\Phi$ in a wide range. Five different values $\Phi$ are considered, equal to $45^{\circ}, 37.5^{\circ}, 30^{\circ}$, and $20^{\circ}$ respectively. Similarly, six different values of cohesion are adopted, equal to $0.01,0.05,0.1,0.2$, and $0.4 \mathrm{~N} / \mathrm{mm}^{2}$ respectively.

In Fig. 8, the failure load of the structure is reported as varying with the mortar cohesion and friction angle. Of course, the failure load varies enormously as the range of the parameters' variation adopted is far too excessive for practical purposes. Nevertheless, this makes it possible to easily identify different possible failure mechanisms and to make interesting observations. Points A, B, C, and D correspond to lower and upper bound assumptions for the cohesion and friction angle. For each of these points, the corresponding deformed shapes at collapse are reported in Fig. 9. As it is possible to note, the different values of mortar friction angle and cohesion determine four different failure mechanisms for the whole structure. In particular, mechanism A corresponds to a combined rocking and shear failure of walls A and B; in mechanism B walls A and B fail in the sliding mode corresponding to the first story only; mechanism $\mathrm{C}$ involves only wall $\mathrm{A}$ with a clear torsional effect of the whole structure; whereas mechanism $\mathrm{D}$ is a combination of the global failure of walls A and B and the out-of-plane overturning of façades 1 and 2 .

The difference between failure mechanisms $\mathrm{A}$ and $\mathrm{B}$ is evident if the deformed shape at collapse of the second story is observed. In fact, in mechanism B the second story rigidly translates, so does not contributing significantly to the ultimate strength of the building, except for a small out-of-plane dissipation mainly concentrated on walls 1 and 2 .

On the other hand, failure mechanisms A and D show that failure is concentrated on walls A and B under in-plane actions. Nevertheless, while walls A and B fail for a combination of rocking and shear in mechanism A (evident in the walls of the first story), they fail for pure shear in mechanism D. The difference is particularly clear if the deformed shape of the elements belonging to the first floor is observed.

Finally, in Fig. 10, the different zones in which the four different mechanisms occur are indicated. It is worth noting that, corresponding to the boundary of each zone, mixed failure mechanisms occur, meaning that for a given set of parameters, the failure mechanism is more difficult to identify, and a moderate change in material parameters can result in different predominant failure mechanisms.

\subsection{D Limit analysis of an ancient masonry building}

In this section, a 3D FE limit analysis on an ancient masonry building is presented. The model is an adaptation of a real house analyzed by De Benedictis et al. in [13] within an extensive survey project of the entire Ortigia (Italy) city center coordinated by Giuffrè [2]. It is worth noting that the same example has been studied by Orduna in [21] by means of a macro-blocks approach and using limit analysis. The building has two storys and it is assumed, for the sake of simplicity, that its plan is rectangular, with dimensions $8.30 \times 5.35 \mathrm{~m}$.

Vertical loading is constituted by the walls' self weight and the permanent and live loads of the first floor and of the roof. The masonry density is assumed equal to $20 \mathrm{kN} / \mathrm{m}^{3}$. Due 

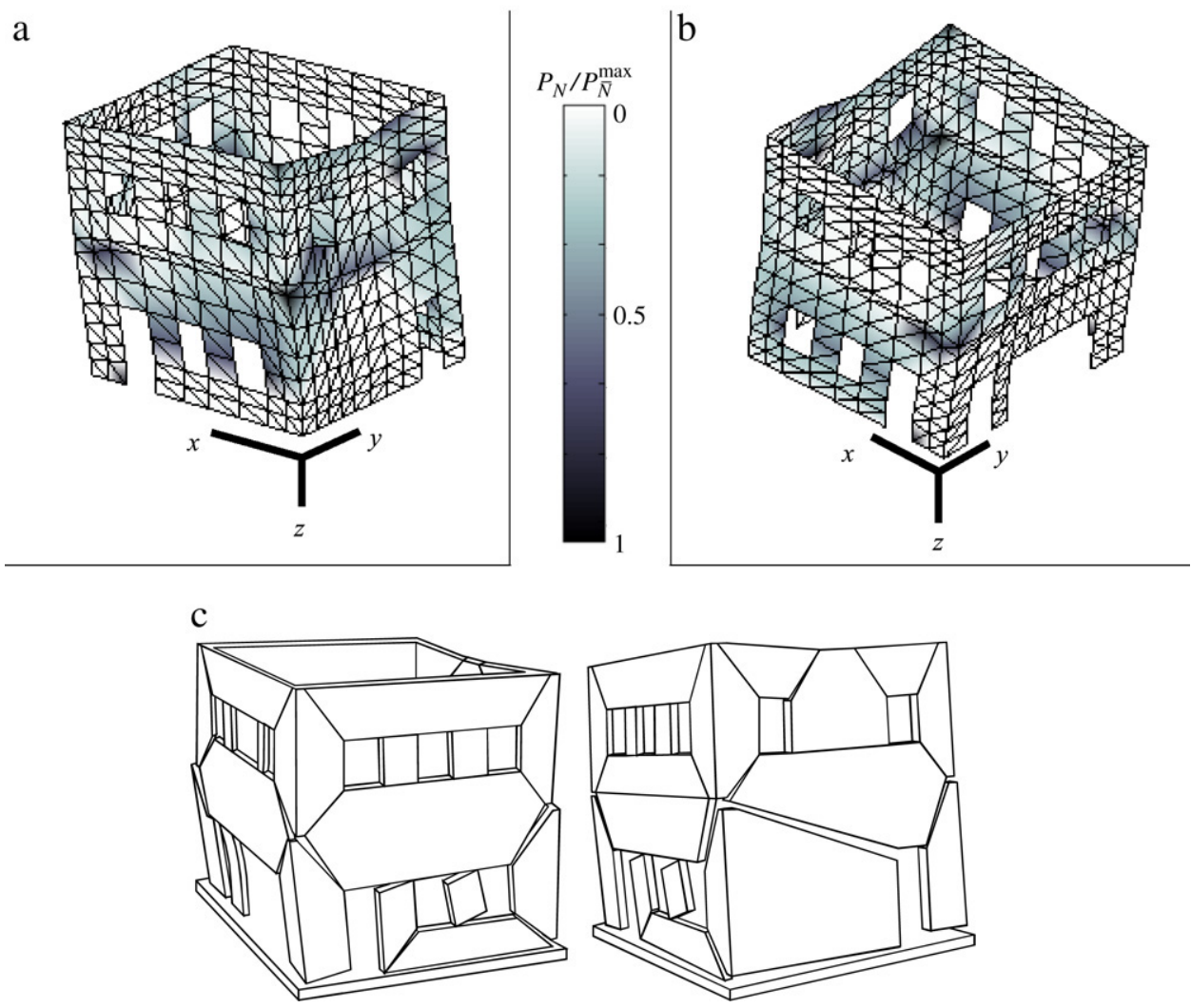

Fig. 7. Yi et al. [12] building. Deformed shape at collapse. a: walls 1-B view. b: walls 2-A view. $P_{N}$ is the in-plane plastic dissipation evaluated at node $N$ and $\bar{N}$ is the node of maximum dissipation. c: Yi et al. [12] deformed shape.

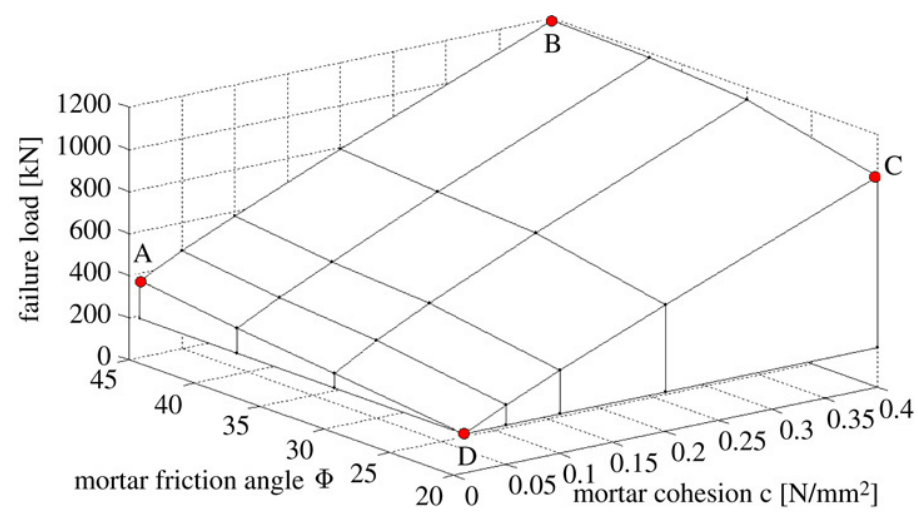

Fig. 8. Yi et al. [12] building. Sensitivity analysis varying mortar cohesion and mortar friction angle.

to the large thickness of the walls, the masonry self weight represents the majority of the total vertical load. The first floor permanent and live loads are equal to $1.61 \mathrm{kN} / \mathrm{m}^{2}$ and $2 \mathrm{kN} / \mathrm{m}^{2}$ respectively. On the other hand, the permanent roof and live loads are equal to $0.87 \mathrm{kN} / \mathrm{m}^{2}$ and $1 \mathrm{kN} / \mathrm{m}^{2}$ respectively. When the seismic load acts, the live loads are reduced by means of a coefficient equal to $1 / 3$.

In Fig. 11, a three dimensional representation of the model is reported. Walls $\mathrm{AB}$ and $\mathrm{DC}$ are assumed to be $60 \mathrm{~cm}$ thick at the first story and $45 \mathrm{~cm}$ at the second story, whereas walls $\mathrm{AD}$ and $\mathrm{BC}$ are $74 \mathrm{~cm}$ and $52 \mathrm{~cm}$ thick respectively. Wall $\mathrm{AD}$ is shared with a contiguous building; consequently only a positive seismic action along $X$ direction is taken into account.
As underlined by De Benedictis et al. [13], the building presents a rocking collapse mechanism of the BC façade, mainly due to the absence of interlocking with its perpendicular walls. Of course, this implies a very low resistance to seismic actions, and an intervention is proposed in [13] in order to improve the interlocking between perpendicular walls and floor's stiffness, so aiming at a global failure mechanism.

In the simulation presented here, the building is taken into consideration only after the intervention proposed in [13]. The intervention provides a new wooden beam floor on the first floor, as well as steel ties at the floor level. Furthermore, the roof structures are strengthened in order to provide in-plane load distribution capacity. 

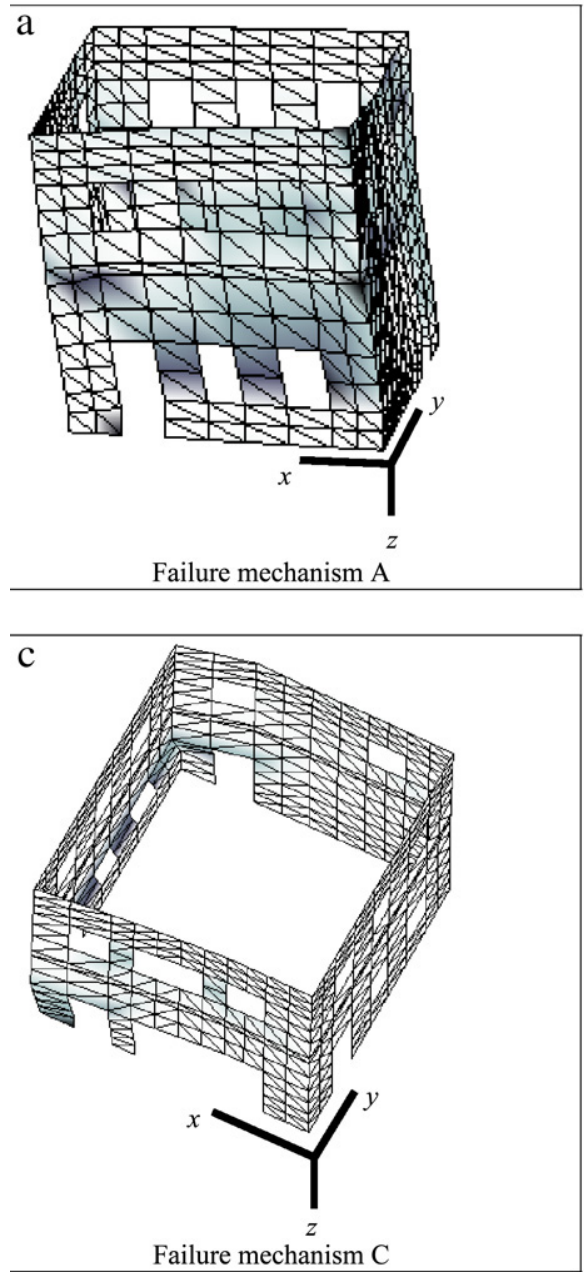
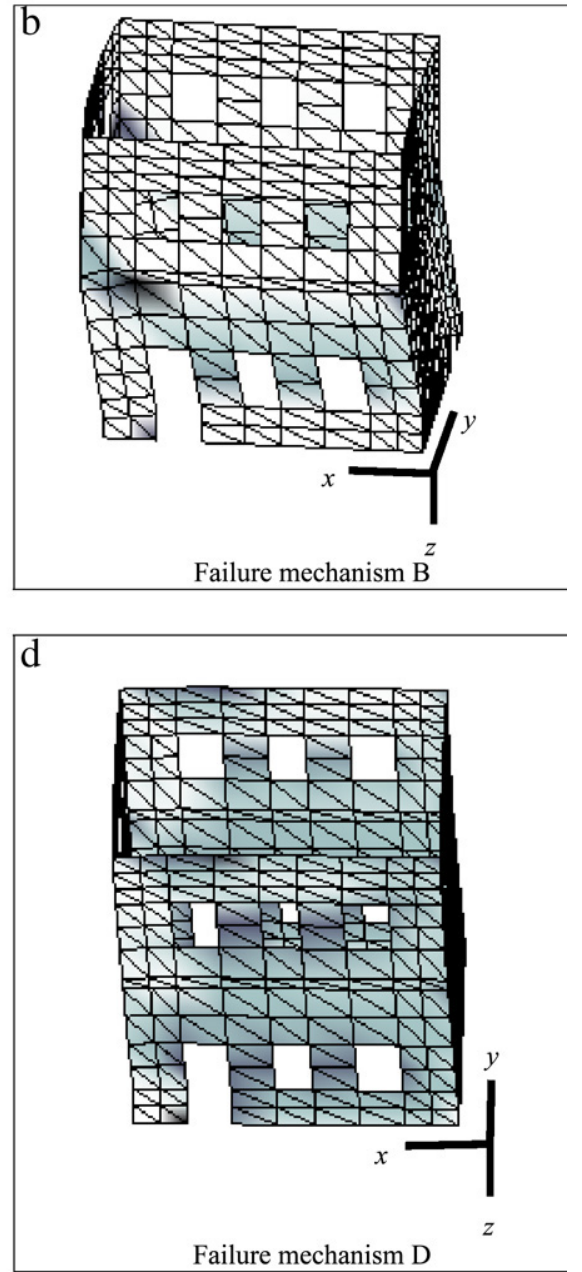

Fig. 9. Yi et al. [12] building. Different failure mechanisms for the structure varying mortar cohesion and friction angle. a. Failure mechanism A. b. failure mechanisms B. c. failure mechanism C. d. failure mechanism D. $P_{N}$ is the in-plane plastic dissipation evaluated at node $N$ and $\bar{N}$ is the node of maximum dissipation.

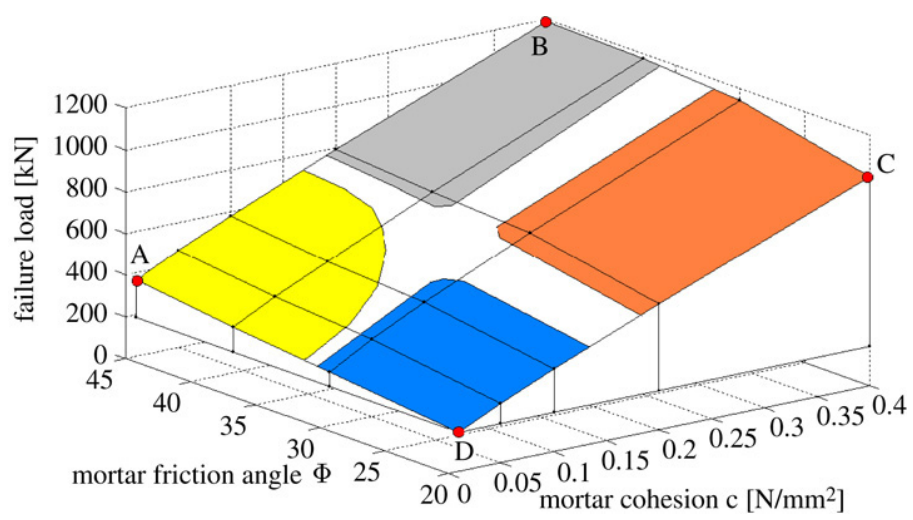

Fig. 10. Yi et al. [12] building. Sensitivity analysis, failure mechanisms patch.

The masonry is supposedly constituted by blocks of dimensions around $46 \times 14 \times 22 \mathrm{~cm}$. In the homogenized FE limit analysis model, for the joints reduced to interfaces a pure Mohr-Coulomb failure criterion with friction angle $\Phi=30^{\circ}$ and cohesion $c=0.01 \mathrm{~N} / \mathrm{mm}^{2}$ is adopted, in order to represent the very low tensile strength of masonry, whereas blocks are supposed to be infinitely resistant. It is stressed that such a value of the friction angle is chosen according to Orduna [21], in absence of precise data available from the literature and in any case in agreement with typical values adopted for the joint.

In the 3D FE limit analysis model, a mesh with 1576 triangular elements is used, as shown in Fig. 12a.

The results obtained with the homogenized FE limit analysis model (i.e. failure shear at the base and failure mechanism) 


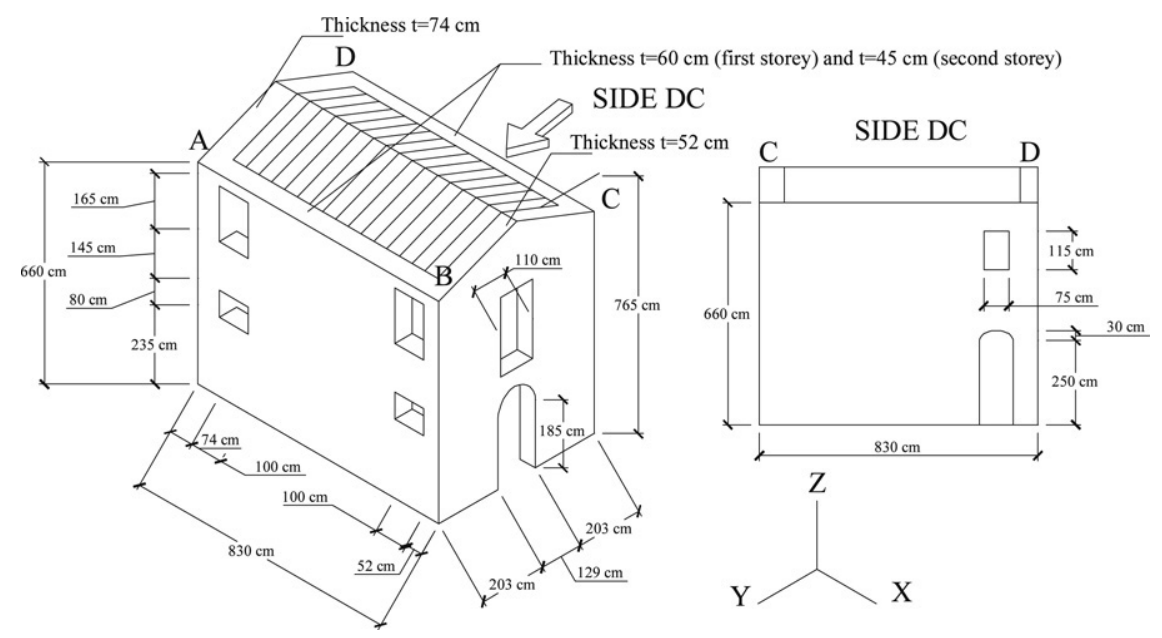

Fig. 11. De Benedictis et al. [13] house, geometry.
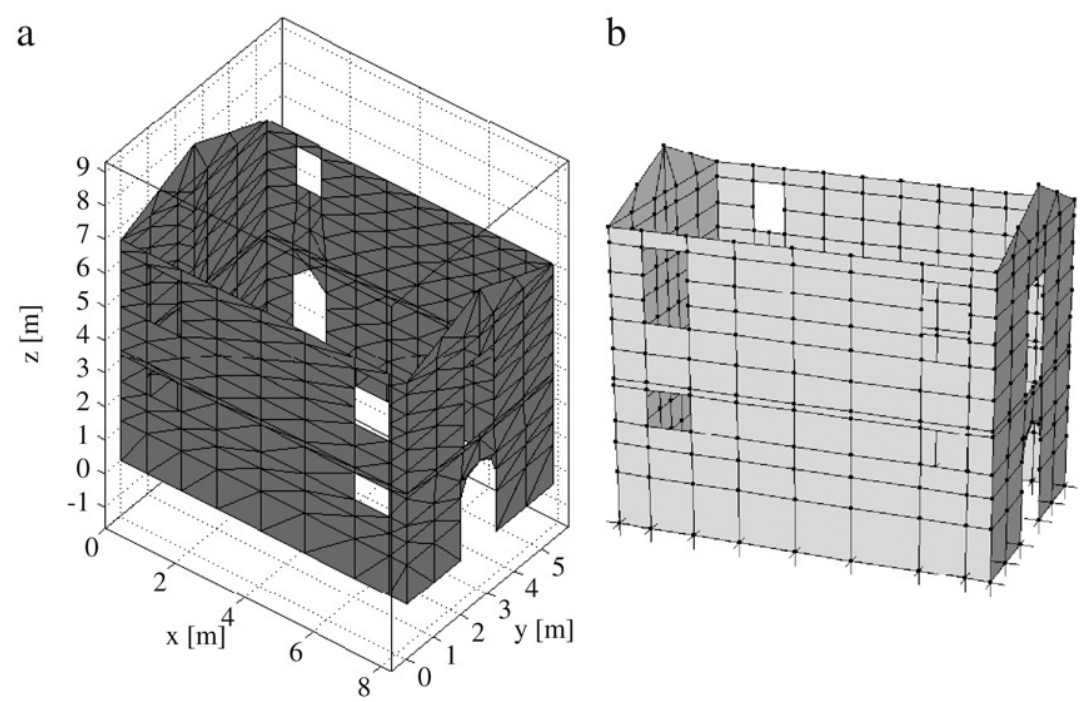

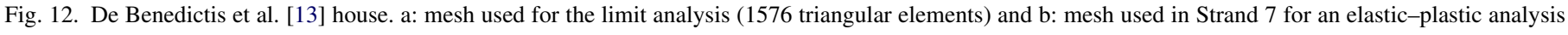
with Mohr-Coulomb failure criterion.

are compared with a standard FE elastic-perfectly plastic analysis performed by means of commercial code, Fig. $12 \mathrm{~b}$. The analysis is conducted using a mesh of 788 four-noded plate elements supposing that the masonry is isotropic with a pure Mohr-Coulomb failure criterion $\left(c=0.01 \mathrm{~N} / \mathrm{mm}^{2}\right.$ and $\Phi=30^{\circ}$ ).

As one can note, an isotropic global Mohr-Coulomb failure criterion strongly differs form a joint Mohr-Coulomb failure criterion, as for instance adopted in this paper, for obtaining masonry macroscopic failure surfaces. In fact, even when an isotropic Mohr-Coulomb failure criterion is chosen for the joints, it has been shown that an orthotropic strength domain for masonry is obtained (see [10] for further details), due to the bricks staggering. As a consequence, masonry strengths along vertical and horizontal directions can differ sensibly and the results are dependent both on the geometry of the elementary cell and on the mechanical properties of mortar.

Thus, isotropic models are not recommended for the analysis of masonry at collapse, since the horizontal strength can significantly differ from the vertical one. Therefore, the comparisons reported here have the sole aim of showing that standard elastic-plastic procedures can fit results obtained with more sophisticated procedures when ad hoc mechanical properties for the isotropic material are adopted. Hence, particular care should be taken by practitioners both in the analysis at collapse of complex masonry structures as well as in the interpretation of the results.

The kinematic FE homogenized limit analysis gives a total shear at the base of the building of $701 \mathrm{kN}$, in good agreement with the results obtained with a standard isotropic FE procedure $(710 \mathrm{kN})$.

In Fig. 13a, the total shear at the base obtained by means of the FE commercial code against the node $N$ displacement (see Fig. 13b) is reported. Furthermore, a comparison between deformed shapes at collapse of both models, Figs. 13b and 14, shows the accuracy of the homogenized model and that the failure is mainly concentrated on wall BC. The opening close to 


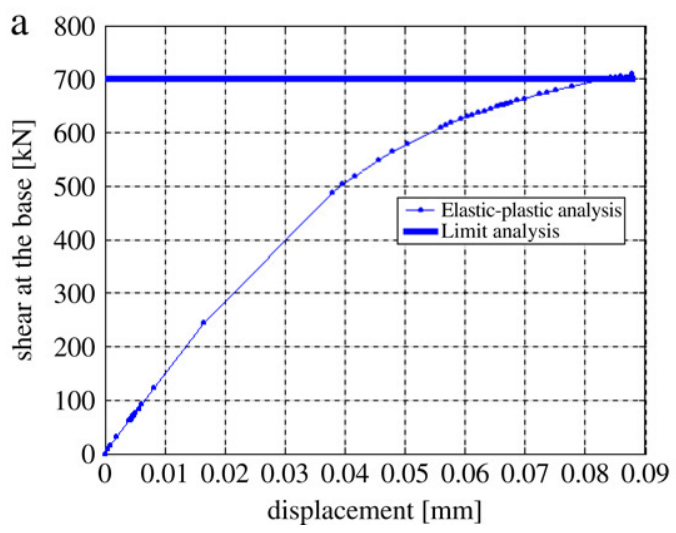

$\mathrm{b}$

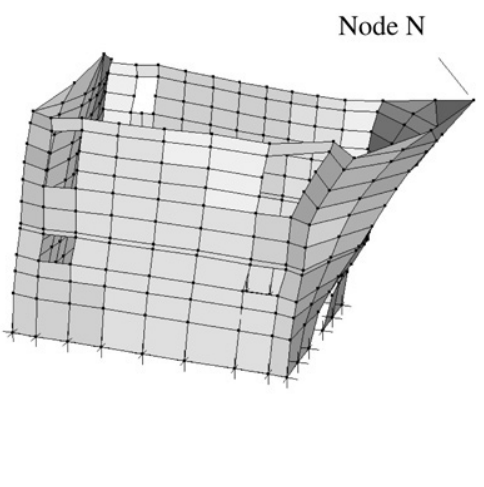

Fig. 13. De Benedictis et al. [13] house. Standard FE elastic-plastic approach. Shear at the base a: node $N$ displacement curve. b: deformed shape at collapse.
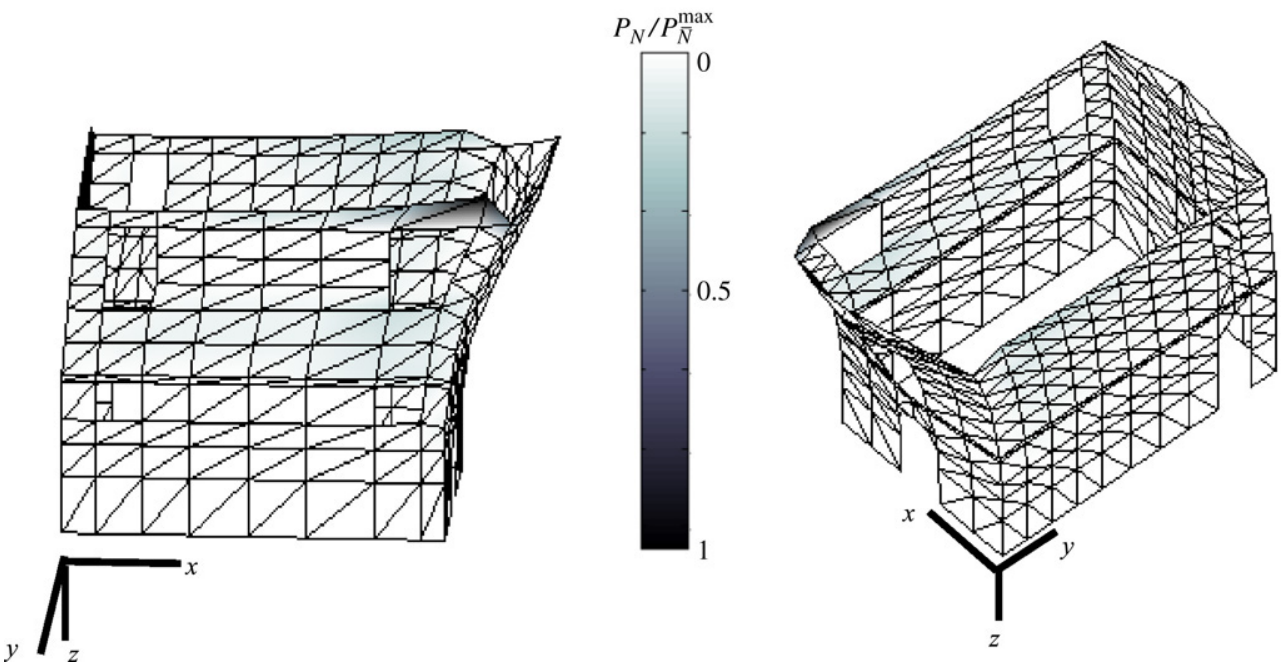

Fig. 14. De Benedictis et al. [13] house. Deformed shape at collapse and concentration of plastic dissipation for the entire building, homogenization FE limit analysis approach. $P_{N}$ is the in-plane plastic dissipation evaluated at node $N$ and $\bar{N}$ is the node of maximum dissipation.

wall $\mathrm{BC}$ at the top of the structure strongly affects the behavior, despite the ties existing at the roof level.

On the other hand, even if a limit analysis gives similar results to a FE elastic-plastic isotropic approach, it cannot prove that either method is accurate or that the predicted failure modes are correct. Nevertheless, it is stressed that the comparison can be useful for practitioners in order to have at their disposal a number of different kinds of information at collapse, such as for instance (a) a reliable prediction of the total shear at the base, (b) plastic dissipation zones, (c) displacements, (d) failure mechanism of the structure, and (e) identification of the more critical zones for possible strengthening, interventions, etc.

Finally, a comparison between the results obtained by means of the homogenization approach proposed here and those obtained with a macro-blocks procedure (see Orduna [21]) is rather interesting. The so called "proportionality coefficients" $\alpha$, defined as the ratios between horizontal loads at failure and vertical loads, are compared. The present homogenization model approximately gives an $\alpha$ equal to 0.36 , whereas with the macro-blocks approach (see [21]) a proportionality coefficient equal to 0.38 is found.
A sensitivity analysis is also conducted for the example at hand. A classic Mohr-Coulomb failure criterion with tension cut-off $f_{t}$ is considered for the joints, assuming $f_{t}$ constantly equal to $\min \left\{0.05 \mathrm{~N} / \mathrm{mm}^{2} c / \tan \Phi\right\}$ and varying the cohesion $c$ and friction angle $\Phi$ in a wide range. Five different values $\Phi$ have been considered, respectively equal to $5^{\circ}, 15^{\circ}, 20^{\circ}, 30^{\circ}$, and $35^{\circ}$. Similarly, six different values of cohesion have been inspected, respectively equal to $0.01,0.063,0.125,0.25,0.375$, and $0.5 \mathrm{~N} / \mathrm{mm}^{2}$.

In Fig. 15, the failure load of the structure is reported, varying the mortar cohesion and the friction angle. It is worth noting that points $\mathrm{A}, \mathrm{B}, \mathrm{D}$, and $\mathrm{E}$ correspond to the lower and upper bound assumptions for cohesion and friction angle, whereas point $\mathrm{C}$ is located as corresponding to the middle of the range inspected. For each of these points, the corresponding deformed shapes at collapse are reported in Fig. 16. As it is possible to note, the different values of the mortar friction angle and cohesion determine four different failure mechanisms for the whole structure. For mechanisms B and C little differences occurred; therefore they are considered equivalent for the sake of simplicity. In particular, mechanisms B and C present a pure façade failure in out-of-plane bending with a vertical yield line 


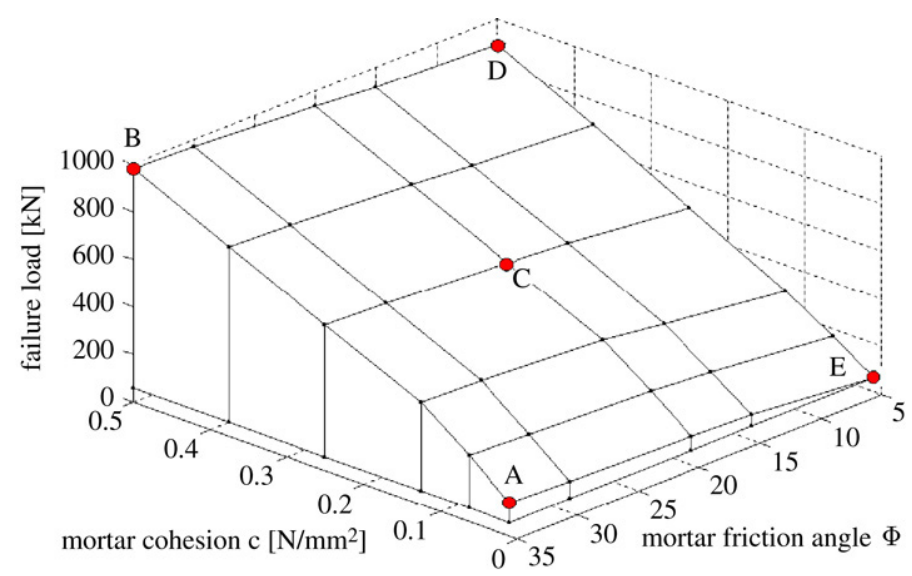

Fig. 15. De Benedictis et al. [13] house. Sensitivity analysis varying the mortar cohesion and the mortar friction angle.

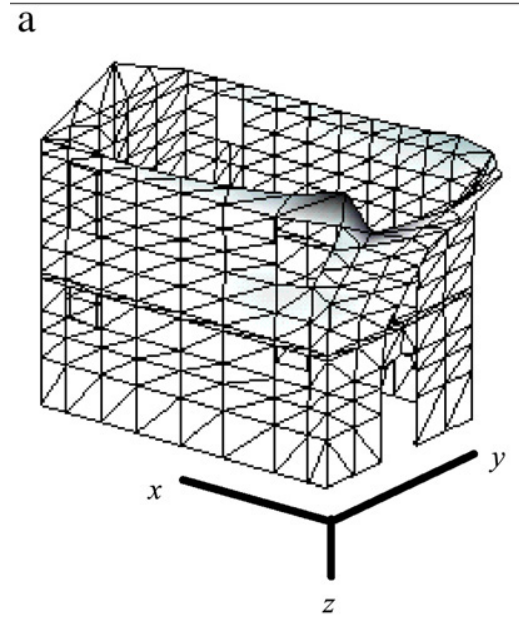

Failure mechanism A

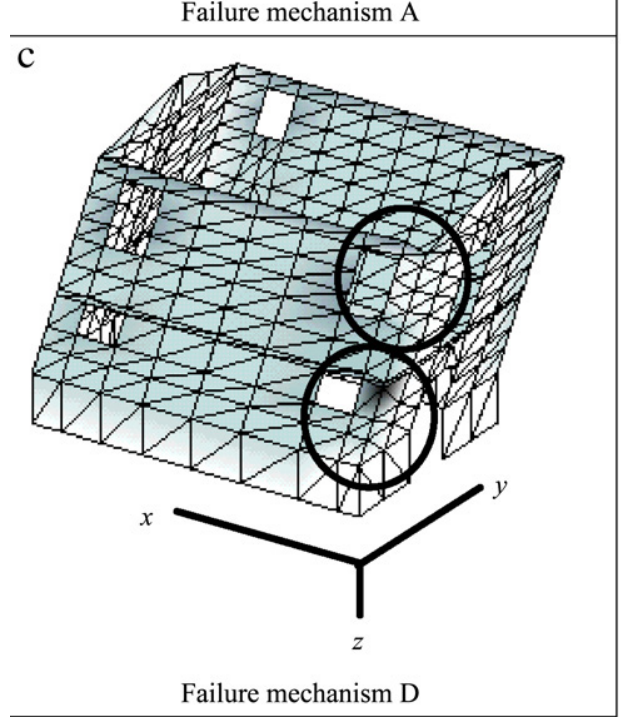

b

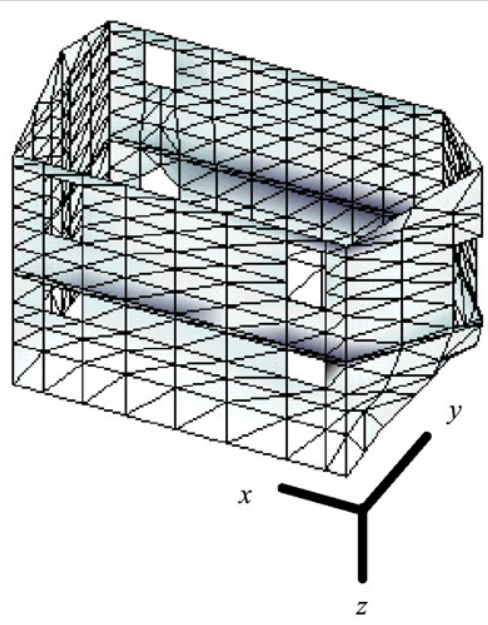

Failure mechanisms B and C

$-0.5$

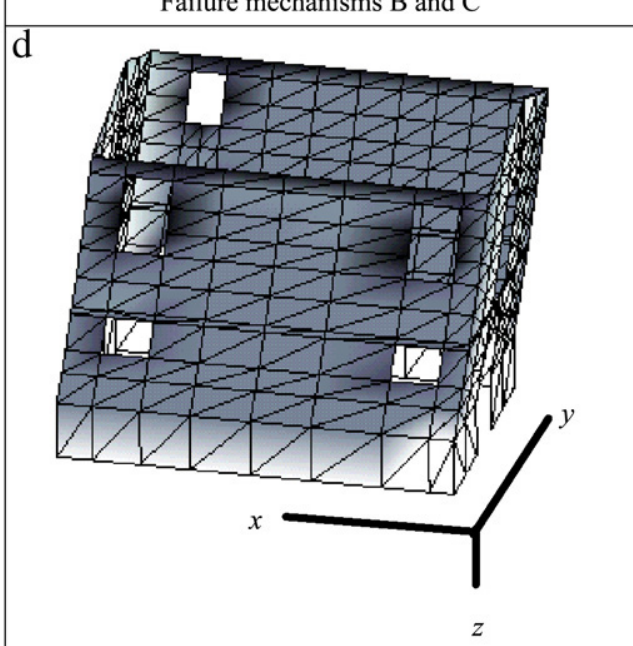

Failure mechanism E

Fig. 16. De Benedictis et al. [13] house. Different failure mechanisms for the structure varying mortar cohesion and friction angle. a: failure mechanism A. b: failure mechanisms B and C. c: failure mechanism D. d: failure mechanism E. $P_{N}$ is the in-plane plastic dissipation evaluated at node $N$, and $\bar{N}$ is the node of maximum dissipation.

at the center of the wall and keeping the edges restrained; mechanism $A$ is a combination of a partial shear failure of walls $\mathrm{AB}-\mathrm{DC}$ and overturning of $\mathrm{BD}$ façade; whereas mechanism
$\mathrm{D}$ involves both walls $\mathrm{AB}$ and $\mathrm{DC}$, which present a typical sliding failure and walls $\mathrm{AC}$ and $\mathrm{BD}$ which overturn with rigid motion around a horizontal hinge at the base of the building. 
a

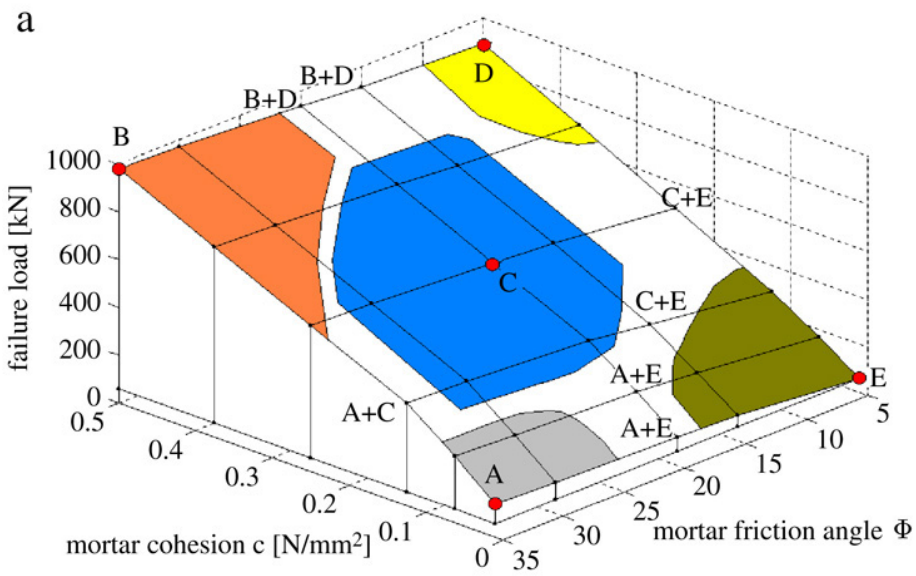

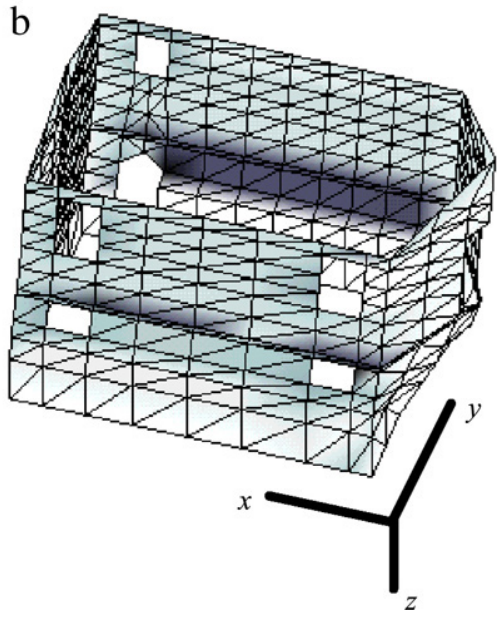

Fig. 17. De Benedictis et al. [13] house. Sensitivity analysis. a: failure mechanisms patch. b: C+E failure mechanism.

Finally, mechanism $\mathrm{E}$ involves both walls $\mathrm{AB}$ and $\mathrm{DC}$ with a clear in-plane shear failure and walls $\mathrm{AC}$ and $\mathrm{BD}$ with an overturning failure. Differently from mechanism $D$, mechanism E provides significant damage nearing the vicinity of the first story openings in walls $\mathrm{AB}$ and DC. A further difference between mechanisms $\mathrm{D}$ and $\mathrm{E}$ relies on the presence of a small zone for mechanism D which fails out-of-plane (see details in Fig. 16c).

Where mechanism A is concerned, failure is mainly concentrated at the second story and involves walls $\mathrm{AB}-\mathrm{DC}$ in a limited region near the corner and corresponding to the opening for wall $\mathrm{AB}$.

It is interesting to note from Fig. 16a and $\mathrm{b}$ that partial out-of-plane collapses of the façades (wall BC) are possible at different values of mortar cohesion and friction angle. In fact, horizontal actions are applied both in correspondence of the floors (with a proportionality coefficient depending of the weight of the floors multiplied by their height) and at the centroids of each element. As a consequence, wall $\mathrm{BC}$ can exhibit large out-of-plane deformations at the middle span, since almost all the vertical weight of the building is represented by masonry self weight, and wood floors are considered deformable in their plane.

Finally, in Fig. 17a the intervals in which the four different mechanisms occur are indicated. As it is possible to note, corresponding to the boundary of each zone, mixed failure mechanisms are present, as for instance the $\mathrm{C}+\mathrm{E}$ failure mechanism reported in Fig. 17b.

\section{Conclusions}

In the present paper, a kinematic FE limit analysis approach for the 3D analysis of masonry buildings subjected to horizontal actions has been presented. Both in- and out-of-plane failures are taken into account in the evaluation of the total internal power dissipated.

Meaningful examples have been treated with the model at hand, and comparisons with standard incremental elastic-plastic procedures have been reported, in order to test the reliability of the homogenized model developed in terms of both collapse mechanism and ultimate shear at the base. The main conclusions are that: (a) the proposed approach allows us to obtain failure mechanisms and collapse loads, provides similar results to more complex approaches based on nonlinear increments and iterative finite element simulations. The results are obtained for a very small fraction of the effort when compared to nonlinear simulations. The maximum processing time of the proposed approach for the examples shown in the present paper does not exceed $150 \mathrm{~s}$; (b) the sensitivity analysis carried out indicates that different predominant failure mechanisms can be obtained in the analysis after a moderate change in the material parameters. Therefore, significant caution is recommended when trying to reproduce existing damage patterns in existing masonry buildings using advanced nonlinear simulations.

\section{Acknowledgments}

P.B. Lourenço gratefully acknowledges the support of the research project POCTI-ECM-61671-2004 from the Portuguese National Science Foundation - Seismic vulnerability reduction of old masonry buildings.

A. Tralli and G. Milani gratefully acknowledge the support of the research project MIUR COFIN 2005 - Resistenza e degrado di interfacce in materiali e sistemi strutturali. Coordinator: Prof. A. Corigliano.

\section{References}

[1] Ramos L, Lourenço PB. Modeling and vulnerability of historical city centers in seismic areas: A case study in Lisbon. Eng Struct 2004;26: 1295-310.

[2] Giuffrè A, editor. Safety and conservation of historical centers: The Ortigia case. Roma-Bari: Laterza Press; 1993 [in Italian].

[3] O.P.C.M. 3274. 20/03/2003. Primi elementi in materia di criteri generali per la classificazione sismica del territorio nazionale e di normative tecniche per le costruzioni in zona sismica [in Italian].

[4] O.P.C.M. 3431/05. 09/05/2005. Ulteriori modifiche ed integrazioni all'OPCM 3274/03 [in Italian].

[5] Lourenço PB, de Borst R, Rots J. A plane stress softening plasticity model for orthotropic materials. Internat J Numer Methods Engrg 1997; 40:4033-57. 
[6] Lourenço PB, Rots JG, Blaauwendraad J. Continuum model for masonry: Parameter estimation and validation. J Struct Engrg, ASCE 1998;124(6): 642-52.

[7] Zucchini A, Lourenço PB. A micro-mechanical model for the homogenisation of masonry. Internat J Solids Structures 2002;39: 3233-55.

[8] Suquet P. Analyse limite et homogeneisation. C R Acad Sci — Series IIB 1983;296:1355-8 [in French].

[9] Milani G, Lourenço PB, Tralli A. Homogenised limit analysis of masonry walls. Part II: Structural examples. Comput Structures 2006;84:181-95.

[10] Milani G, Lourenço PB, Tralli A. Homogenised limit analysis of masonry walls. Part I: Failure surfaces. Comput Structures 2006;84:166-80.

[11] Milani G, Lourenço PB, Tralli A. A homogenization approach for the limit analysis of out-of-plane loaded masonry walls. ASCE J Struct Engrg 2006;132(10):1650-63.

[12] Yi T, Moon FL, Leon RT, Khan LF. Lateral load tests on a twostory unreinforced masonry building. ASCE J Struct Engrg 2006;132(5): 643-52.

[13] De Benedictis R, de Felice G, Giuffrè A. Safety and conservation of historical centres: The Ortigia case. In: Giuffrè A, editor. Seismic retrofit of a building. 1991. p. 189-217 [chapter 9].
[14] Yi T, Moon FL, Leon RT, Khan LF. Analyses of a two-story unreinforced masonry building. ASCE J Struct Engrg 2006;132(5):653-62.

[15] Sloan SW, Kleeman PW. Upper bound limit analysis using discontinuous velocity fields. Comput Methods Appl Mech Engrg 1995;127(1-4): 293-314.

[16] Munro J, Da Fonseca AMA. Yield-line method by finite elements and linear programming. J Struct Engrg ASCE 1978;56B:37-44.

[17] Cannarozzi AA, Capurso M, Laudiero F. An iterative procedure for collapse analysis of reinforced concrete plates. Comput Methods Appl Mech Engrg 1978;16:47-68.

[18] Krabbenhoft K, Lyamin AV, Hjiaj M, Sloan SW. A new discontinuous upper bound limit analysis formulation. Internat J Numer Methods Engrg 2005;63:1069-88.

[19] Sloan SW. A steepest edge active set algorithm for solving sparse linear programming problems. Internat J Numer Methods Engrg 1988;12:61-7.

[20] Olsen PC. Rigid-plastic finite element analysis of steel plates, structural girders and connections. Comput Methods Appl Mech Engrg 2001;191: 761-81.

[21] Orduna A. Seismic assessment of ancient masonry structures by rigid blocks limit analysis. Ph.D. thesis. Portugal: University of Minho; 2003. Available at: http://www.civil.uminho.pt/masonry. 